\title{
Studying the influence of moving vehicle on air pollutant dispersion through environmental chamber
}

\author{
T.T. Chow ${ }^{1, *}$, Wenjing Zhang ${ }^{1}$, and Jinliang Wang ${ }^{2}$ \\ ${ }^{1}$ Division of Building Science and Technology, City University of Hong Kong, Hong Kong SAR, China \\ ${ }^{2}$ School of Petroleum Engineering, Changzhou University, Jiangsu, China
}

\begin{abstract}
The risk of air pollutants like particulate matters on human beings has been widely reported. One main concern is its health impact on people through direct emission or resuspension. In China with the quick growth in private car ownership, the worries about the influence of the moving vehicles on particulate dispersion is growing. In this study, the influence of a moving object on wick formation and particulate dispersion was investigated. An advanced numerical model was developed, in which the unsteady Eulerian RANS model was applied for simulating the airflow, the modified drift-flux model for modelling particulate dispersion, and the dynamic mesh model for mimicking the moving vehicle. The results show that the vehicular movement induces three noticeable vortexes around the moving body, and the faster the running speed, the stronger the secondary airflow generated.
\end{abstract}

\section{Introduction}

The presence of gaseous pollutants and particulate matters (PM) is linked with many health issues such as cardiovascular diseases, asthma, cardiopulmonary death and lung cancer etc., as well as the transmission of infectious diseases [1]. Life expectancy is casually related to community average fine particle pollution concentrations. With the quick growth of the private car ownership, many people in China are worrying about the deleterious impact of $\mathrm{CO}, \mathrm{NO}_{\mathrm{X}}$ and $\mathrm{PM}_{2.5}$ on the pedestrians due to the moving vehicles. So far, the use of advanced CFD techniques to simulate the influence of moving vehicle on such pollutant dispersion was rarely reported. In this study, the effects were investigated through both experimental and numerical analyses.

\section{Numerical modelling}

\subsection{Dynamic airflow model}

Although DNS (Direct Numerical Simulation) and LES (Large Eddy Simulation) can generally obtain more detailed turbulent information and higher accuracy for turbulent airflow under the influence of object movement, the computational time, cost and required hardware resources can be very demanding and unacceptable. In practice, the RANS (Reynolds-Averaged Navier-Stokes) method can be a good alternative. In our study, the unsteady standard k- $\varepsilon$ turbulence model was adopted to simulate the dynamic airflow [2] under the influence of vehicular movement. The general governing equation, including continuity, momentum, turbulent kinetic energy $k$ and turbulent dissipation rate $\varepsilon$, can be written as:

$$
\frac{\partial(\rho \phi)}{\partial t}+\nabla \cdot(\rho \phi \vec{V})=\nabla \cdot\left(\Gamma_{\phi} \nabla \phi\right)+S_{\phi}
$$

where $\rho$ is the air density; $\vec{V}$ is the velocity vector; $\phi$ represents respectively each of the three velocity components $u, v, w$, the turbulent kinetic energy $k$ as well as the turbulent dissipation rate $\varepsilon ; \Gamma_{\phi}$ is the effective diffusion coefficient for each dependent variable, and $S_{\phi}$ is the source term.

\subsection{Pollutant dispersion model}

To simulate the particle dispersions in a confined space, an Eulerian-Eulerian based modified drift-flux model (MDFM) was adopted. This was encoded into the FLUENT platform [3] through a user-defined function. When applying the Eulerian-Eulerian based MDFM, the one-way coupling assumption is made because of the very low particle volume fraction. This means that the airflow phase can only influence the transport of particles phase but not vice versa. Moreover, the particle concentration can be obtained directly. The governing equation of MDFM for one specific particle group size is then: $\frac{\partial C^{+}}{\partial t}+\nabla \cdot\left[\left(\vec{V}+\vec{v}_{s}\right) \cdot C^{+}\right]=\nabla \cdot\left[\left(D+\varepsilon_{p}\right) \cdot \nabla C^{+}\right]+S_{c}$

where $C^{+}$is the dimensionless particle concentration defined as the ratio of particle number concentration to particle number concentration released at the air supply

\footnotetext{
* Corresponding author: bsttchow@cityu.edu.hk
} 
opening; $\vec{V}$ and $\vec{v}_{s}$ are the velocity of air and gravitational settling of particles respectively; $D$ is the Brownian diffusivity of particles; $\varepsilon_{p}$ is the particle turbulent diffusivity; and $S_{c}$ is the particle source term.

For the treatment of the particle concentration source term $S_{c}$, the entire particle concentration field is divided into two zones: the particle concentration core zone and the near-wall particle concentration boundary layer. In the core zone $S_{c}$ is taken as zero. In the boundary layer $S_{c}$ is considered as the particle concentration sink term, which can be derived from the particle wall deposition flux in the first near-wall cell. The particle wall deposition flux can be formulated as:

$$
J_{\text {cell }}=v_{d, \text { cell }} \cdot C_{b, c e l l}^{+}
$$

where $v_{d, \text { cell }}$ is the particle deposition velocity in nearwall cell.

For those chemically stable gaseous pollutants, the Species Transport Model is capable of predicting its mixing and transport. FLUENT predicts the local mass fraction (Y) of the target species through the solution of a convection-diffusion equation as follows:

$$
\frac{\partial}{\partial t}(\rho Y)+\nabla \cdot(\rho \vec{v} Y)=-\nabla \cdot \vec{J}
$$

where $\mathrm{J}^{\rightarrow}$ is the mass diffusion flux of the target species due to the concentration gradients.

\subsection{Dynamic mesh model}

The dynamic mesh model has been adopted to simulate the dynamic airflow and pollutant concentration field, where the geometrical shape of the concerned domain is changing with time. The integral form in general can be expressed as:

$$
\frac{d}{d t} \int_{V} \rho \phi d V+\int_{\partial V} \rho \phi\left(\vec{u}-\vec{u}_{g}\right) \cdot d \vec{A}=\int_{\partial V} \Gamma \nabla \phi \cdot d \vec{A}+\int_{V} S_{\phi} d V
$$

where $\rho$ is the airflow density, the general scalar $\phi$ represents respectively the different dependent variables of airflow in equation (1) and the dimensionless particle concentration in equation (2); $\vec{u}$ is the airflow velocity vector, $\vec{u}_{g}$ is the grid velocity of the moving mesh, $\Gamma$ is the diffusion coefficient corresponding to different scalar $\phi, S_{\phi}$ is the source term, and $\partial V$ represents the boundary of the control volume.

\section{Dynamic performance evaluation}

\subsection{Case study}

The following describes the numerical treatment of single bus movement in relation to the laboratory test set-up. The computational domain is divided into two separate zones: the 'dynamic' mesh zone and the 'static' mesh zone (Fig. 1). As the object moves forward, only the meshes within the dynamic mesh zone will change with time and object position. The interface data exchange between the two zones is implemented by the grid interface principles of the sliding mesh theory. The dynamic layering method is employed to update mesh, where the grid cell layers adjacent to the moving boundaries are expanded or split according to the specified height of the gird layer. The user-defined functions are programmed to define and track the vehicular motion.

During the iterative procedure, the governing equations are discretised on finite-volumes. The SIMPLE algorithm is applied to solve the pressure-velocity coupling equations. The default Standard Scheme is used for the pressure corrective equation. For the discretization of the governing equations, the Second-order Upwind Scheme is employed for the convection terms and the Central Differences Scheme with second-order accuracy for the diffusion terms. For unsteady modelling, the time derivatives are discretised using the First-order Implicit Scheme.

\subsection{Laboratory measurements}

Most related model validation works done previously and reported in literatures were conducted based on steady state measurements [4]. In this study, further validations were carried out to evaluate the capability of the integrated numerical model applying to dynamic simulation. Laboratory measurements were conducted in an environmental chamber for a downscale model of a double-decker bus, which was triggered to move on a straight-line path. The motion was programmed, and in principle by starting with steady acceleration, then travelling at a cruising speed, and finally decelerating

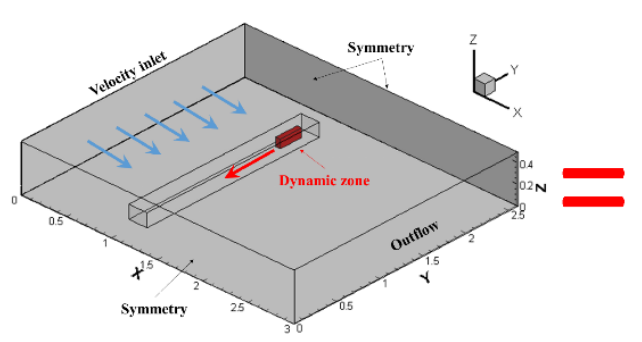

Computational domain

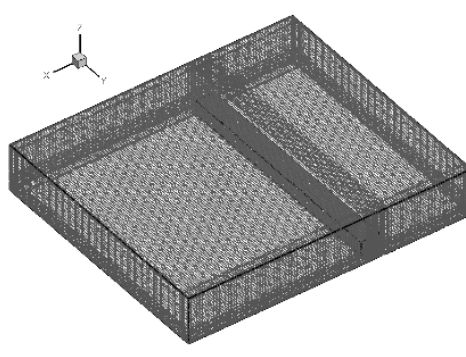

Static mesh zone

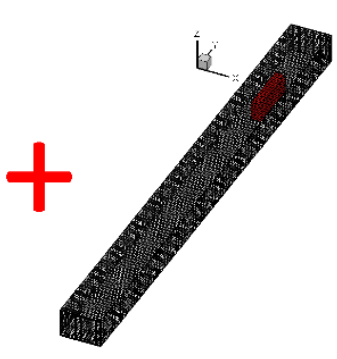

Dynamic mesh zone

Fig. 1. Configuration of the integrated computational domain. 
steadily back to stationary at the end of the track (Fig. 2). The bus model was mounted on the top of an electrical slider (Fig. 3) with the moving speed remotely controlled by a computer system.

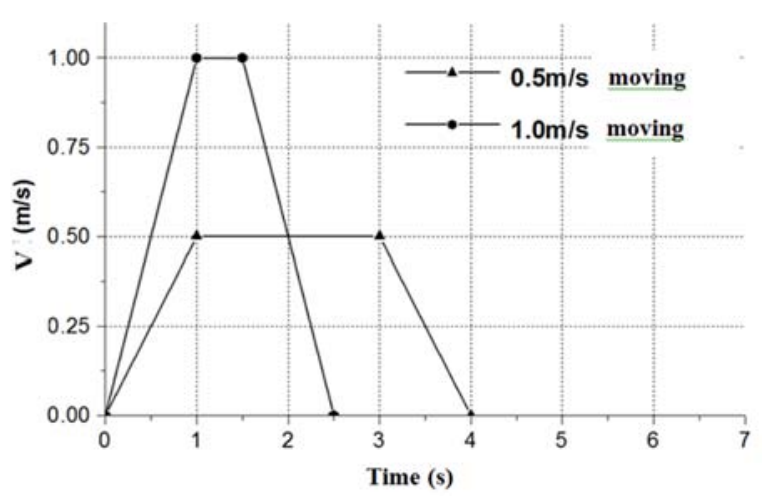

Fig. 2. Examples of vehicle speed setting.

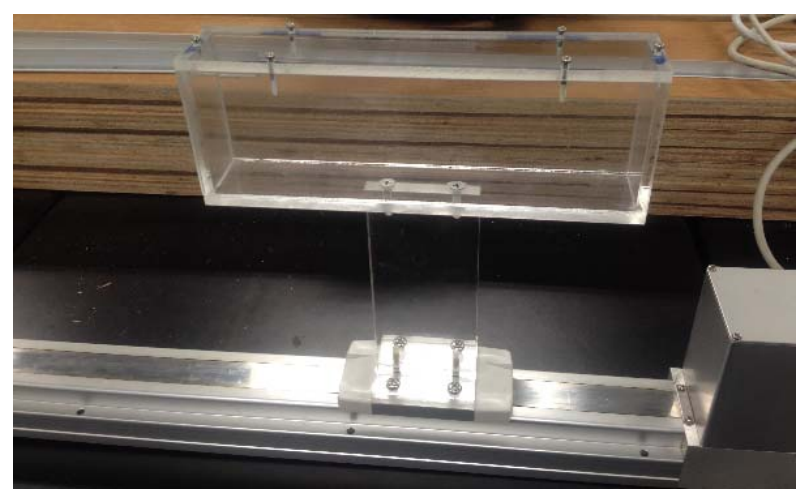

Fig. 3. Electric actuator with the bus model mounted on the sliding plate.

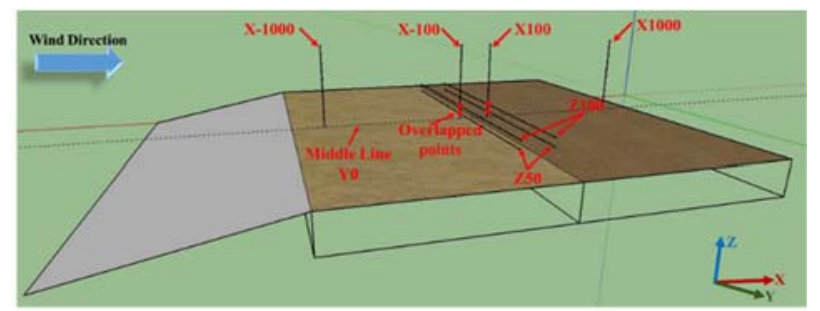

Fig. 4. Physical layout of the vehicle moving track and sampling positions.

A model-mounted tracer-gas supply and discharge system was developed to model the emission from the moving object. A long and pliable plastic tube was used to connect the gas cylinder and the exhaust tailpipe on the vehicle model. For the measurement of airflow velocity, the SWEMA omnidirectional anemometer was employed. The tracer gas concentration was monitored by the HFR400 fast flame ionization detector system. On other occasions, PM2.5 particles can be continuously released from air supply openings at a controlled release concentration with the dispersion picked up by the particle concentration counter installed. The airflow inside the environmental chamber was taken as isothermal, with the approaching wind perpendicular to the vehicle travelling path. Fig. 4 shows the physical layout located with the sampling positions.

\subsection{Model validation}

The numerical predictions of both the mean (timeaveraged) and the transient (time-dependent) airflow velocity and tracer gas concentration were checked against the measurements from the stationary tests and the dynamic tests as well. Both the horizontal and vertical distributions of the mean flow velocity measured in the stationary tests were found reasonably predicted through computation. The time-average magnitude of the instantaneous flow velocity at different sampling sites were also compared. In doing so, the instantaneous velocity was normalized by the mean velocity in the initial steady state, giving the non-dimensional velocity (Vel*). As shown in Fig. 5, the good matching between the measured and modelled Velocity* clearly demonstrated the capability of the Standard k- $\varepsilon$ Model coupled with the Dynamic Mesh Model to provide a reasonably accurate representation of the transient flow physics under the dynamic influence of vehicular motion. Moreover, good matching was also confirmed in the prediction of timevarying tracer gas concentration.

\section{Discussion and conclusion}

In our analysis, the comparison of velocity contours, flow streamlines, and pollutant concentration contours were first made by observing the steady-state and transientstate solutions, in order to qualitatively capture the general characteristics of the dynamic impact. Then, the transient variations of the instantaneous velocity and tracer gas concentration at various monitoring sites were analysed. The results show that the motion induces three noticeable vortexes around the moving vehicle - the faster the travelling speed, the stronger the induced secondary airflow. At specific monitoring point, peak velocity and particulate concentration was detected at a short instant after the vehicle model passed through that point. Then afterwards, the pollutant dispersion pattern will return to the normal situation and will be governed by the bulk airflow.

The "propelling effect", the "wake effect" and the "recovery process" were determined as the three basic mechanisms of the vehicle-induced dynamic impact (Fig. 6), which depends on the ambient airflow, the object movement, as well as the receptor position relative to the travelling path. Strong fluctuations of air velocity and relatively high tracer gas concentrations can be witnessed in the regions close to the travelling path, but such dynamic impact faded off with distance. The ambient air was pushing away as the vehicle running forward, resulting in an acceleration zone with high airflow velocity at the front. Such "propelling effect" was suppressed by the strong approaching wind but was enhanced by the vehicle running at fast speed. At the same time, the surrounding air was entrained to generate the 
"wake effect", which was intensified by stronger wind and lower vehicle speed. Hence receptors close to the travelling path are prone to high exposure risks under the condition of calm wind and slow vehicle motion.

Apart from the above useful observations together with the advanced model development, the way to quantify the resuspension characteristics of inhalable particles deposited on ground surfaces remains challenging. Our direction has been on obtaining instantaneous resuspension rate correlations and considering this in the source term of MDFM by converting the instantaneous resuspension rate into an instantaneous resuspension flux. Further experimental works need to be done to help in the model development.
The work described in this article was financially supported by the research grants of the City University of Hong Kong (Strategic Research Project no. 7004795).

\section{References}

1. A.P. Jones, Atmos. Environ. 33, 4535 (1999)

2. T.T. Chow, J.L. Wang, Build. Environ. 57, 68 (2012)

3. FLUENT user's guide. https://www.sharcnet.ca/ Software/Fluent6/html/ug/main pre.htm

4. E. Solazzo, X. Cai, S. Vardoulakis, 2008. Atmos. Environ. 42, 4918 (2008)
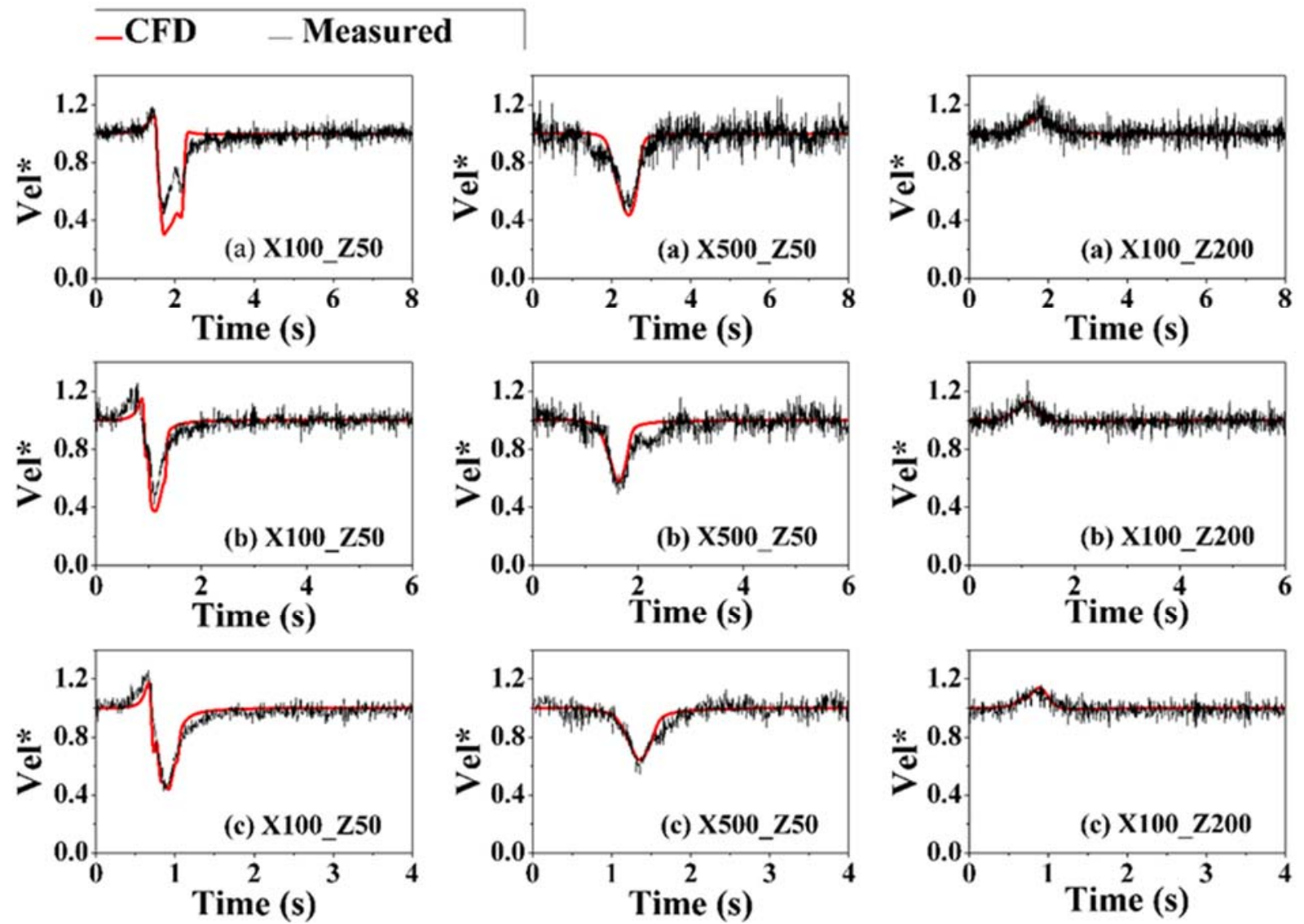

Fig. 5. Time series of the normalized instantaneous velocity $\left(\mathrm{Vel}^{*}\right)$ modelled and measured at different monitoring sites along the center of the moving path (Y0) in different speed modes: (a) slow, (b) moderate, and (c) fast.

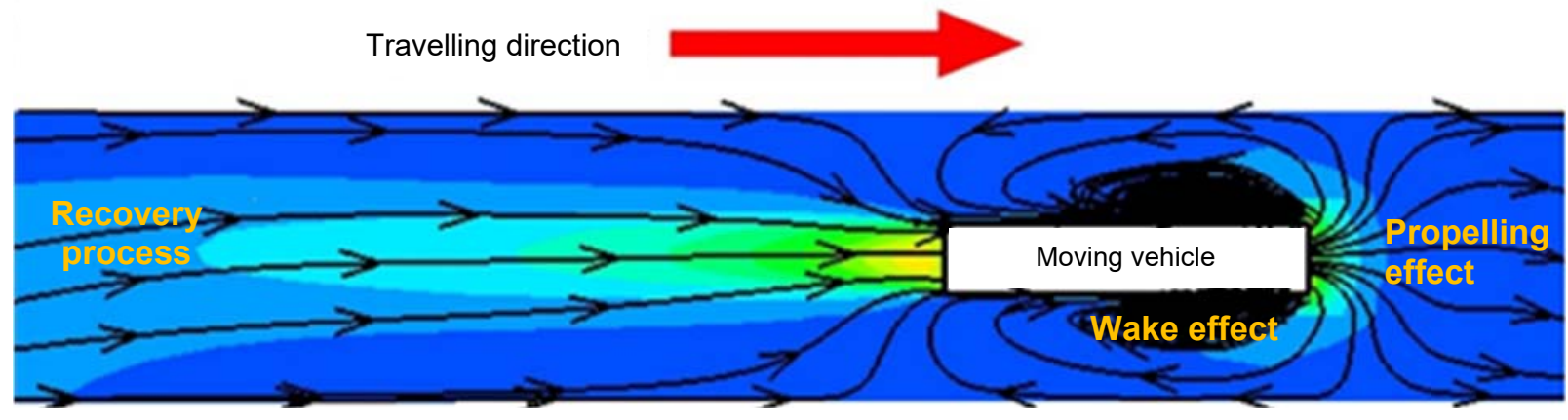

Fig. 6. Airflow streamlines around a moving vehicle at the height of adult breathing zone. 